\title{
Article \\ Self-Start Multi-Wavelength Laser Source with Tunable Delay-Line Interferometer and Optical Fiber Reflector for Wireless Communication System
}

\author{
Amare-Mulatie Dehnaw ${ }^{1}$, Run-Kai Shiu ${ }^{1}$, Ruei-Bin Chen ${ }^{1}$, Jyun-Wei Li ${ }^{1}$, Yibeltal-Chanie Manie ${ }^{1}$, \\ Hsing-Chih Liang ${ }^{2}$ and Peng-Chun Peng ${ }^{1, *}$ \\ 1 Department of Electro-Optical Engineering, National Taipei University of Technology, Taipei 10608, Taiwan; \\ t108999404@ntut.edu.tw (A.-M.D.); oo6401@yahoo.com.tw (R.-K.S.); lux111679@gmail.com (R.-B.C.); \\ t109659001@ntut.edu.tw (J.-W.L.); yibeltal_chanie@ntut.edu.tw (Y.-C.M.) \\ 2 Institute of Optoelectronic Science, National Taiwan Ocean University, Keelung 20224, Taiwan; \\ hcliang@mail.ntou.edu.tw \\ * Correspondence: pcpeng@ntut.edu.tw; Tel.: +886-2-2771-2171 (ext. 4671)
}

\section{check for}

updates

Citation: Dehnaw, A.-M.; Shiu, R.-K.; Chen, R.-B.; Li, J.-W.; Manie, Y.-C.; Liang, H.-C.; Peng, P.-C. Self-Start Multi-Wavelength Laser Source with Tunable Delay-Line Interferometer and Optical Fiber Reflector for Wireless Communication System. Appl. Sci. 2021, 11, 9553. https:// doi.org/10.3390/app11209553

Academic Editors: Christos Verikoukis and Christos Bouras

Received: 21 July 2021

Accepted: 11 October 2021

Published: 14 October 2021

Publisher's Note: MDPI stays neutral with regard to jurisdictional claims in published maps and institutional affiliations.

Copyright: (c) 2021 by the authors. Licensee MDPI, Basel, Switzerland. This article is an open access article distributed under the terms and conditions of the Creative Commons Attribution (CC BY) license (https:/ / creativecommons.org/licenses/by/ $4.0 /)$.

\begin{abstract}
The radio-over-fiber (RoF) technique has gained a lot of interest recently, as the millimeterwave signals can be generated and delivered in the optical domain with the advantages of low attenuation, high capacity, and being free from electromagnetic noise interference (EMI). In this paper, we propose and experimentally prove a self-start multi-wavelength laser source based on a distributed feedback laser diode (DFB-LD) for the RoF transport system. The self-start multiwavelength laser source generates stable laser power with less than $0.18 \mathrm{~dB}$ power fluctuation and exhibits good stability. In order to estimate the transmission performance, data is externally modulated onto the multi-wavelength by a reflective semiconductor optical amplifier (RSOA) and transmitted through single-mode fiber (SMF). The experimental result proves that the proposed RoF transport system achieves error-free transmission and clear eye diagrams.
\end{abstract}

Keywords: radio-over-fiber; multi-wavelength laser; wireless communication system

\section{Introduction}

Performance-boosting of bandwidth and long-distance coverage are currently the most pressing concerns for end-to-end connection and quality of service in both wired and wireless broadband access [1-5]. Thus, the goals of transmitting and receiving more data as enhanced Mobile Broadband (eMBB), interconnecting millions of devices at once by means of massive machine type communication (mMTC), and increasing responsiveness by ultra-reliability and low latency communication (URLLC) for fifth-generation (5G) new radio (NR) next-generation communication systems are considerably studied [6-13]. The radio-over-fiber (RoF) technique, which can generate and deliver millimeter-wave signals in the optical domain, is a good candidate for next-generation communication systems due to the advantages of fiber such as high capacity, low attenuation, lightweight, and being free from electromagnetic noise interference (EMI) [14-18]. In the RoF transmission system, the desired signal can be optically upconverted to a higher frequency band by means of selecting the specific wavelength. Hence, the multi-wavelength generation technique plays an important role in the RoF system. Conventional multi-wavelength schemes use an array of laser sources, external modulators, and local oscillators. However, adding multiple devices in the central station increases the overall cost of the transmission system. In [19-21], semiconductor optical amplifiers (SOAs) and erbium-doped fiber amplifiers (EDFAs) are utilized as gain mediums to generate multi-wavelengths. Although EDFA has the characteristics of a flatter gain spectrum, lower polarization-dependent gain, and higher saturation power, it has a strong line broadening and cross-saturation at room temperature. Hence, it is challenging to secure stable multi-wavelength lasing at room temperature using 
EDFA. In [22], an SOA-based ring laser using coupled ring cavities is used to generate multi-wavelength. However, its performance is limited by polarization, and it is difficult to control the cavity.

Optoelectronic oscillators (OEOs) have attracted much attention owing to the characteristics of high-quality factors, extremely low noise, excellent performance even at higher frequencies, and other characteristics that are not achieved with electronic oscillators. Conventional OEO schemes require external modulators [23-25] and continuous-wave lasers. However, an external modulator causes high insertion loss due to its low modulation efficiency. Therefore, the conventional OEO scheme needs three electrical amplifiers [23].

In this research, a self-start multi-wavelength laser source is proposed and experimentally demonstrated, achieved by directly modulating a commercially available distributed feedback laser diode (DFB-LD) without any local oscillator. The self-start multi-wavelength laser source is based on a self-starting optoelectronic oscillator [23]. A double-pass filter composed of an optical circulator, tunable delay line interferometer (TDLI), and an optical fiber reflector (OFR) is proposed to enhance the tone-to-noise ratio (TNR). A tunable filter (OTF) is used to choose wavelengths for baseband or wireless signal. A reflective semiconductor optical amplifier (RSOA) modulates data signals and is sent across a $25 \mathrm{~km}$ single-mode fiber (SMF). The system performance is verified using clear eye diagrams and error-free signal transmission.

\section{Experimental Setup and Results}

The schematic structure of the proposed self-start multi-wavelength laser source for wireless networks is shown in Figure 1. The demultiplexer selects two wavelengths from the multi-wavelength laser source for each channel [17]. The desirable data streams are modulated onto the selected wavelengths by the RSOA. Then, the multiplexer combines all the wavelengths before the SMF transmission. After fiber transmission, the demultiplexer splits the received optical signal into different base stations (BS) and a photodetector (PD) is employed to convert the optical signal into an electrical signal before being emitted by the antenna for wireless transmission.

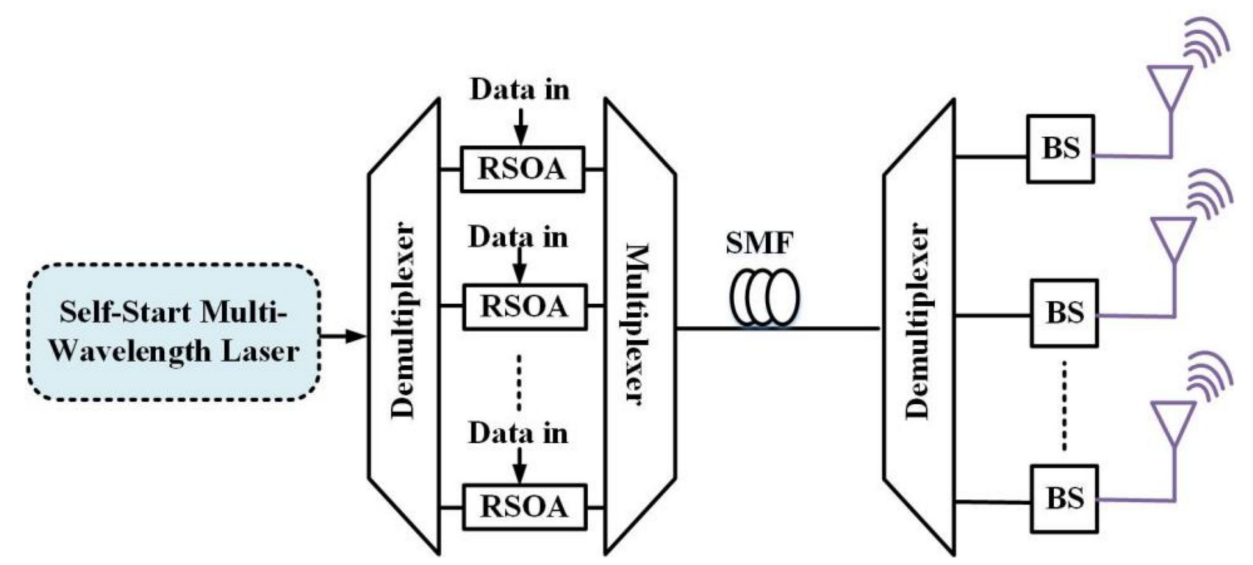

Figure 1. Schematic illustration of the self-start multi-wavelength laser for RoF transport systems for wireless applications (RSOA: reflective semiconductor optical amplifier, BS: base station, and SMF: single-mode fiber).

The detailed configuration of the proposed self-start multi-wavelength laser source is depicted in Figure 2. The lightwave emitted from the DFB laser is launched to port 1 of the optical circulator. Then, the lightwave output from port 2 of the optical circulator $(\mathrm{OC})$ is routed to a tunable delay interferometer and reflected back to the OC by the optical fiber reflector. Subsequently, port 3 of the OC passes the lightwave to a $1 \times 2$ optical coupler with a $90 / 10$ ratio. The branch with a $10 \%$ power ratio is connected to the optical spectrum analyzer for the monitor, while the other branch sends the lightwave to a PD (PP10G) for optical-to-electrical conversion. The electrical signal is amplified by an electrical 
amplifier (EA), filtered by a high-Q bandpass filter (BPF), and then inserted into the DFB laser for direct modulation after being amplified again by the second EA. After several rounds of the above-mentioned operation, the self-start multiwavelength laser based on the OEO structure is generated. In the optical path, TDLI is employed to reshape the multi-wavelength, and the OFR reflects the lightwave back to the TDLI for the second reshaping to boost the tone-to-noise ratio (TNR). In the electrical path, the first EA is utilized to compensate for the loss caused by the optical-to-electrical conversion, and the second EA is used to boost the electrical signal before being fed into the DFB. The 3-dB bandwidth of the high-Q BPF is $10 \mathrm{MHz}$, and the $\mathrm{Q}$ factor is calculated to be 995.3 .

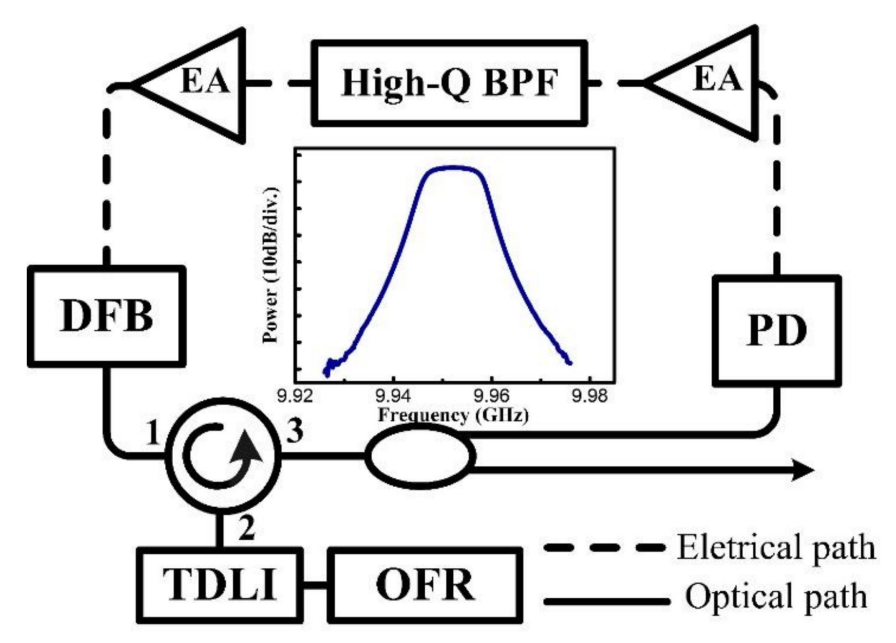

Figure 2. Experimental setup of OEO-based multi-wavelength generation (DFB: distributed feedback laser, TDLI: tunable delay interferometer, OFR: optical fiber reflector, PD: photodetector, EA: electrical amplifier, High Q BPF: High-Q bandpass filter).

The radio-frequency (RF) tone generated by the OEO structure is measured by the electrical analyzer as illustrated in Figure 3. By adjusting the gain of the second EA, the output power of the RF tone can be tuned to modulate the DFB laser for manipulating the output number of the wavelength of the self-start multi-wavelength laser source. As demonstrated in the inset of Figure 3, the numbers of multiwavelength are 3, 5, and 10 when the power of the inserted RF tone is $6 \mathrm{dBm}, 10 \mathrm{dBm}$, and $20 \mathrm{dBm}$, respectively.

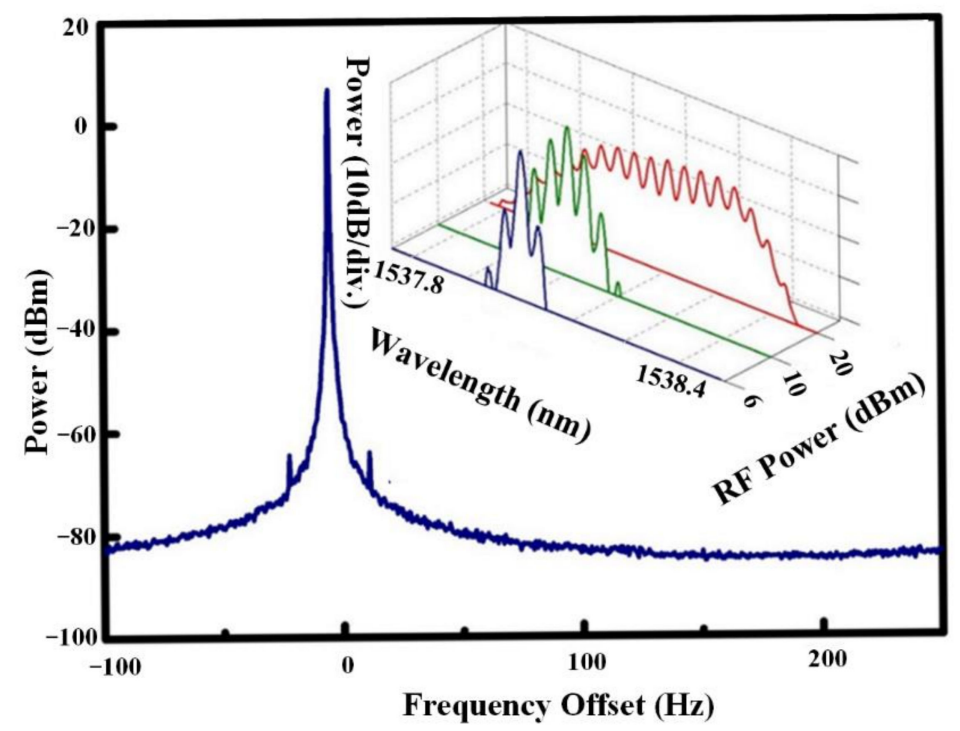

Figure 3. A generated RF signal's output spectrum and laser output vs. RF power. 
When the power of RF tone inserted into the DFB laser increases, the number of the generated multi-wavelength increases. In contrast to the number of wavelengths, the tone-to-noise ratio (TNR) decreases. Therefore, to enhance the TNR, the TDLI is deployed to reshape the multi-wavelength. Figure 4 demonstrates the measured spectra and the measuring setup of two different structures of TDLI. For the first structure, OSA captures the optical spectrum of the broadband light source after passing the TDLI for single filtering, as the red line illustrates in Figure 4. The signal-to-noise ratio (SNR) of single filtering is only $10 \mathrm{~dB}$. To enhance these SNR results, a second structure with double filtering is proposed. The broadband light source is launched to port 1 of OC and then routed to the TDLI for first filtering.

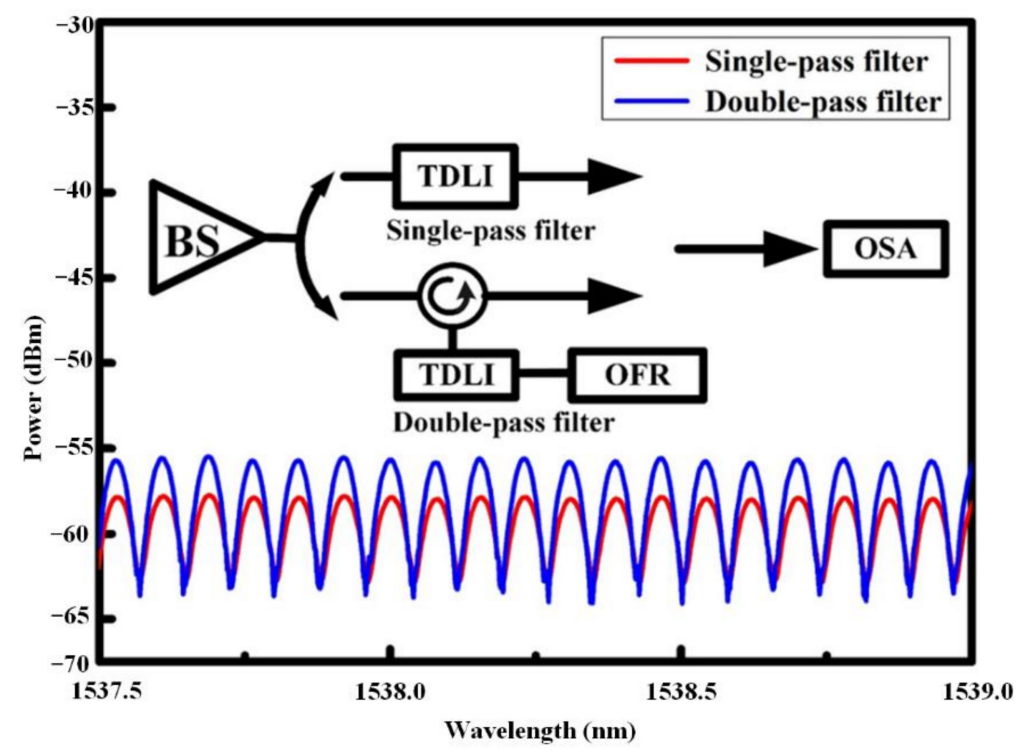

Figure 4. The broadband source with single-pass and double-pass filters.

The filtered broadband light source is reflected by the OFR and inserted back to the TDLI again for second filtering. After, the lightwave output from port 3 of OC is measured by the OSA, as the red line shows in Figure 4.

Compared to single filtering, the double filtering structure, whose SNR is $16 \mathrm{~dB}$, exhibits better performance. A round trip through the same tunable delay interferometer reduces the linewidth of the transmission peaks and is effective in suppressing spontaneous noise. The optical spectra of the self-start multi-wavelength laser, with and without the double filtering technique, are demonstrated in Figure 5. Without double filtering, the power fluctuation is about $7.5 \mathrm{~dB}$ and the TNR is $7 \mathrm{~dB}$. By employing double filtering, the TNR has been improved to $18 \mathrm{~dB}$.

As the stability of the self-start multi-wavelength laser is another important factor, the optical power of $1537.768 \mathrm{~nm}, 1537.848 \mathrm{~nm}, 1537.928 \mathrm{~nm}, 1538.004 \mathrm{~nm}, 1538.084 \mathrm{~nm}$, $1538.16 \mathrm{~nm}, 1538.24 \mathrm{~nm}, 1538.32 \mathrm{~nm}, 1538.396 \mathrm{~nm}$, and $1538.476 \mathrm{~nm}$ are chosen to be monitored every 2 min as illustrated in Figure 6. Among all wavelengths, the power fluctuation is less than $0.18 \mathrm{~dB}$, showing good stability of the proposed scheme.

The experimental configuration of the RoF transport system based on the proposed self-start multi-wavelength laser is illustrated in Figure 7. In the central office, the multiwavelength laser is externally modulated by the data via RSOA. At the transmission end, we use semiconductor optical amplifiers as modulators, in the output spectrum current region of RSOA is between $60 \sim 80 \mathrm{~mA}$; we also fixed the RSOA bias current at $70 \mathrm{~mA}$ as an application. Subsequently, the modulated lightwave is amplified by an EDFA before the OTF. The OTF is utilized to select the specific wavelength to allocate the RF frequency. After $25 \mathrm{~km}$ SMF transmission, the PD converts optical components into an electrical signal and up-converts the signal to a higher frequency band based on the wavelength spacing of 
the selected wavelength at the BS. The optical signals displayed on OSA and the electrical signal were transmitted to the electrical spectrum analyzer and bit error tester.

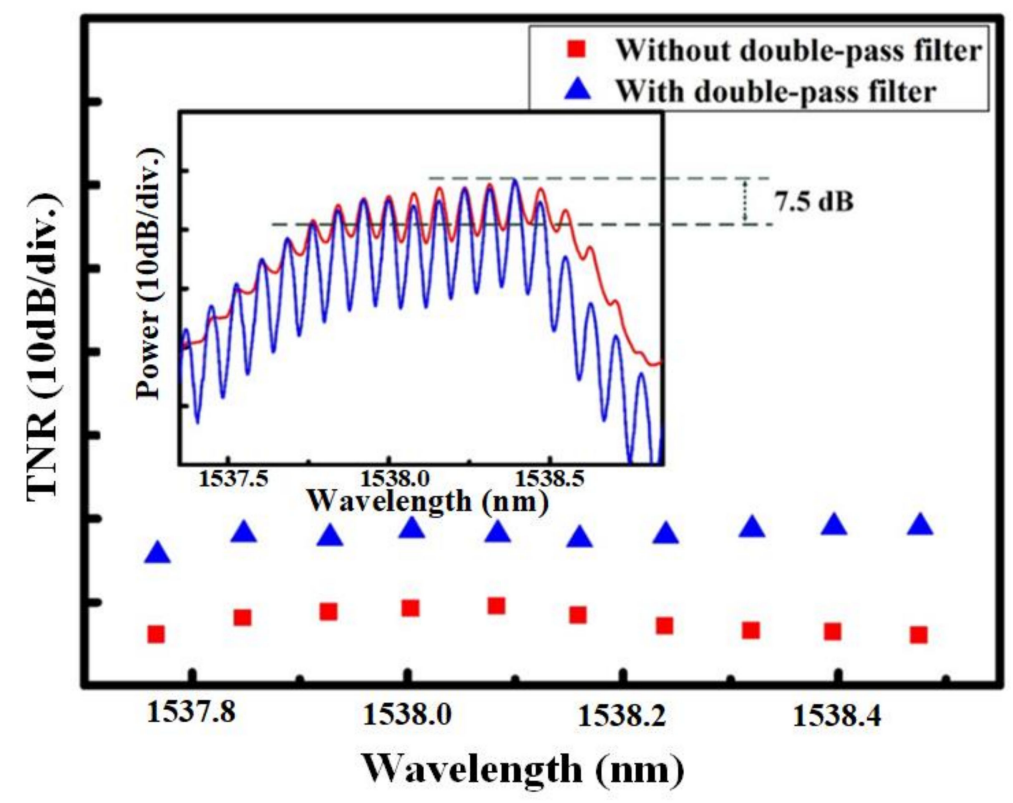

Figure 5. Output optical and TNR spectrum with and without a double-pass filter.

The bit error rate (BER) curve and eye diagram of $155 \mathrm{Mbps}$ and $622 \mathrm{Mbps}$ signals after back-to-back (BTB) within $25 \mathrm{~km}$ SMF distance data transmissions are illustrated in Figure 8, where we selected only one wavelength optical spectrum of the multi-wavelength laser. Figure 8 shows a BER and eye diagram with different modulation data. As demonstrated in Figure 8, the eye diagrams are clear and open. The eye diagrams of $155 \mathrm{Mbps}$ and $622 \mathrm{Mbps}$ signals were captured at $-22.5 \mathrm{dBm}$ and $-21.5 \mathrm{dBm}$, respectively. The received optical power is measured using power monitor-attenuators (EigenLight Corporation). The measured point is before the PD. The table in Figure 8 shows the power penalty in different data rates. The power penalty of $155 \mathrm{Mbps}$ and $622 \mathrm{Mbps}$ signals were $0.1 \mathrm{~dB}$ and $0.2 \mathrm{~dB}$, respectively. The power penalty for $622 \mathrm{Mbps}$ signal transmission was relatively higher due to the effect of fiber dispersion.

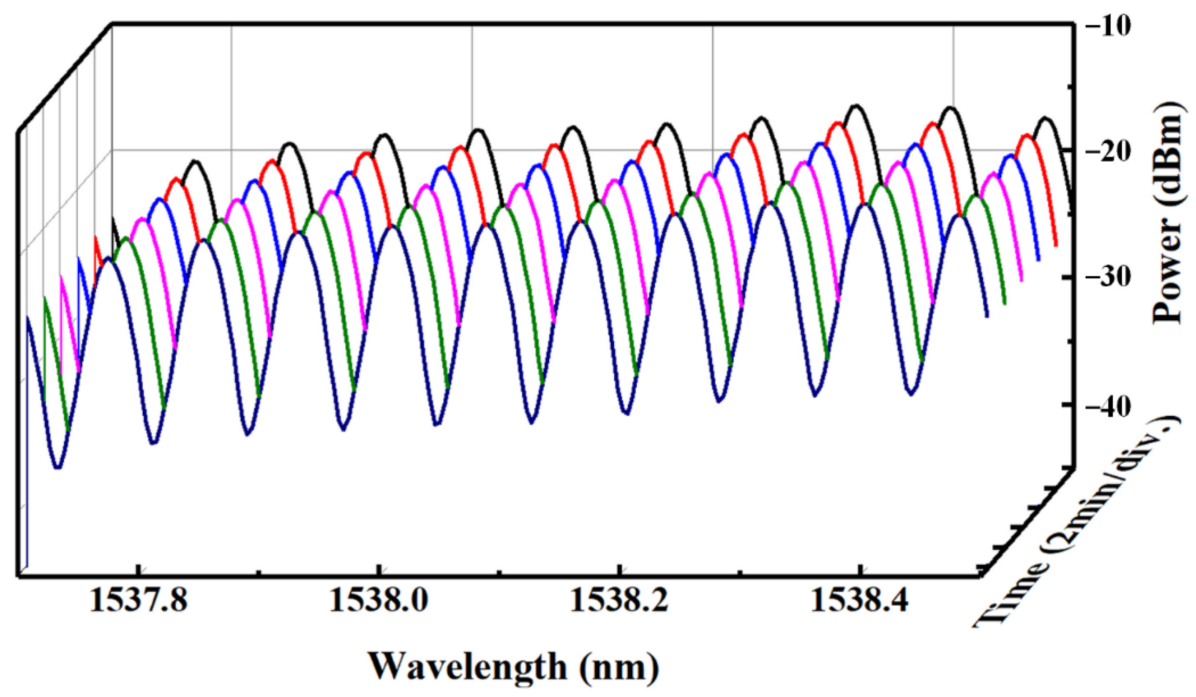

Figure 6. Cont. 

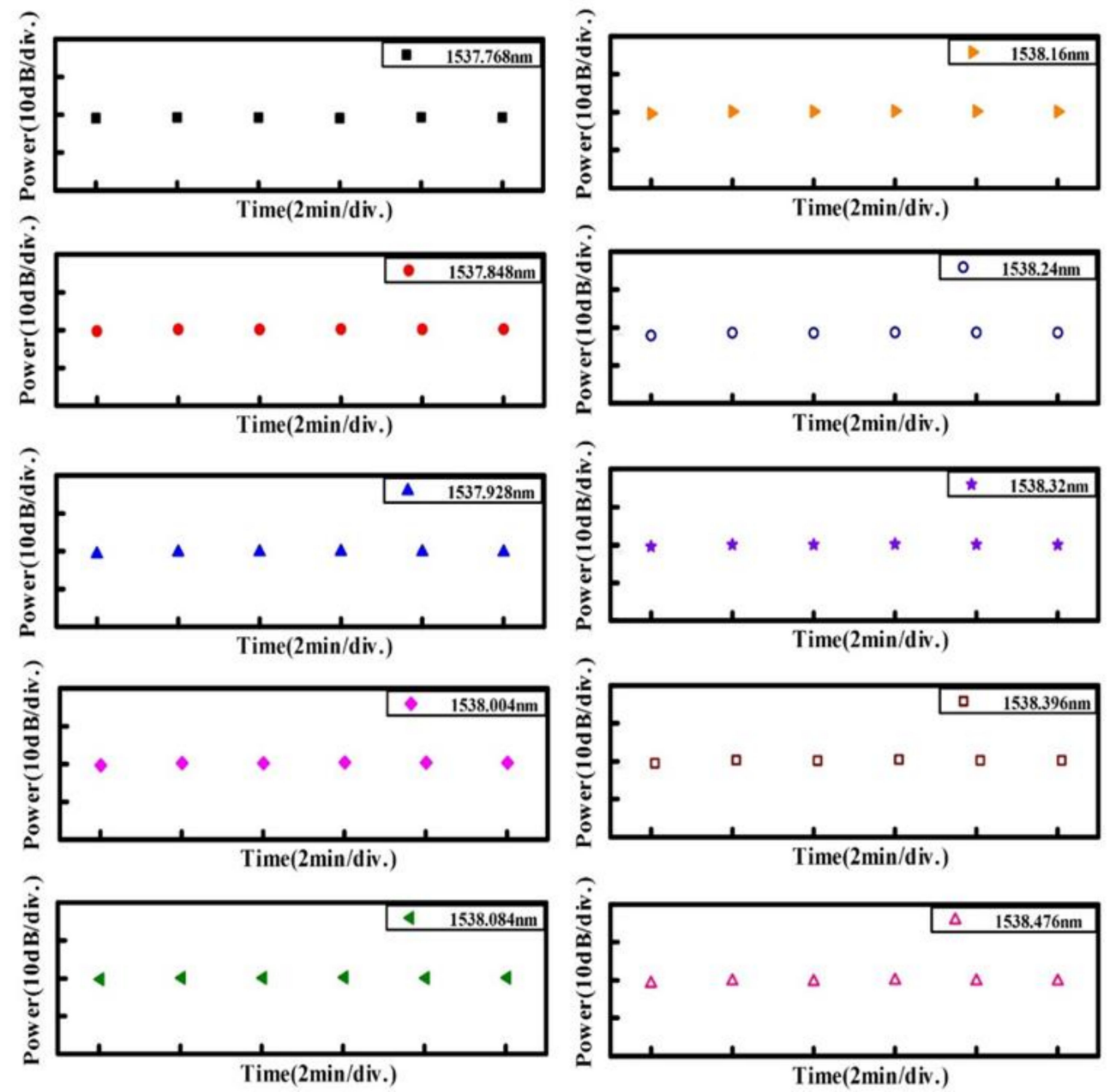

Figure 6. Output power stability for the selected wavelengths.

The optical spectra of the multi-wavelength laser and the selected two wavelengths are illustrated in the top inset of Figure 9. The wavelength spacing of the two selected wavelengths is about $0.08 \mathrm{~nm}$, and thus the $155 \mathrm{Mbps}$ data carried by these two optical tones is upconverted to $9.953 \mathrm{GHz}$. The bottom inset of Figure 9 demonstrates the measured electrical spectrum of the $155 \mathrm{Mbps}$ data. The center frequency of the signal is located at $9.953 \mathrm{GHz}$ and the eye diagram is also clear. The RF signal is generated by the two-tone interference method [17]. The SNR is over $20 \mathrm{~dB}$, which may support future wireless broadband access applications. Moreover, the eye diagram was captured at $-16.5 \mathrm{dBm}$. The frequency of the RF signal depends on the central frequency of the high-Q BPF; we can use different central frequencies of the high-Q BPF for different applications [24]. To further analyze the performance of the system, the bit error rate versus different optical received power was measured. Error-free is achieved while the optical received power is larger than $-16.5 \mathrm{dBm}$.

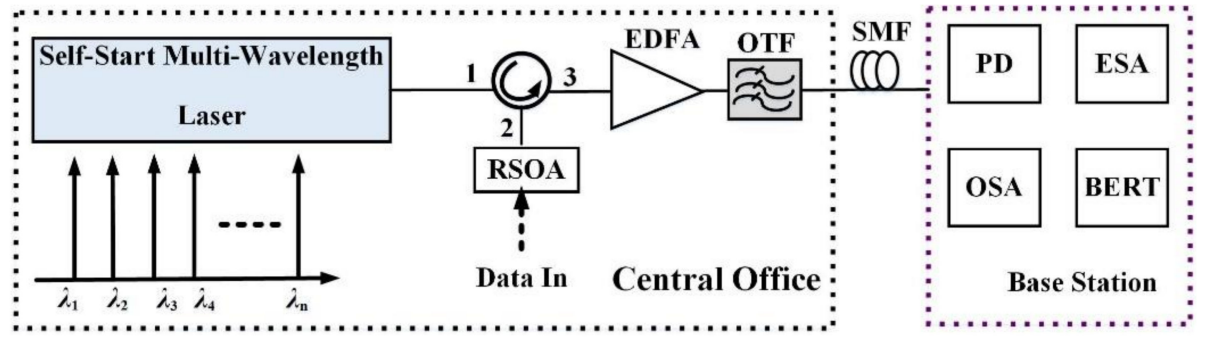

Figure 7. The architecture of the self-start multi-wavelength laser for RoF transmission. 


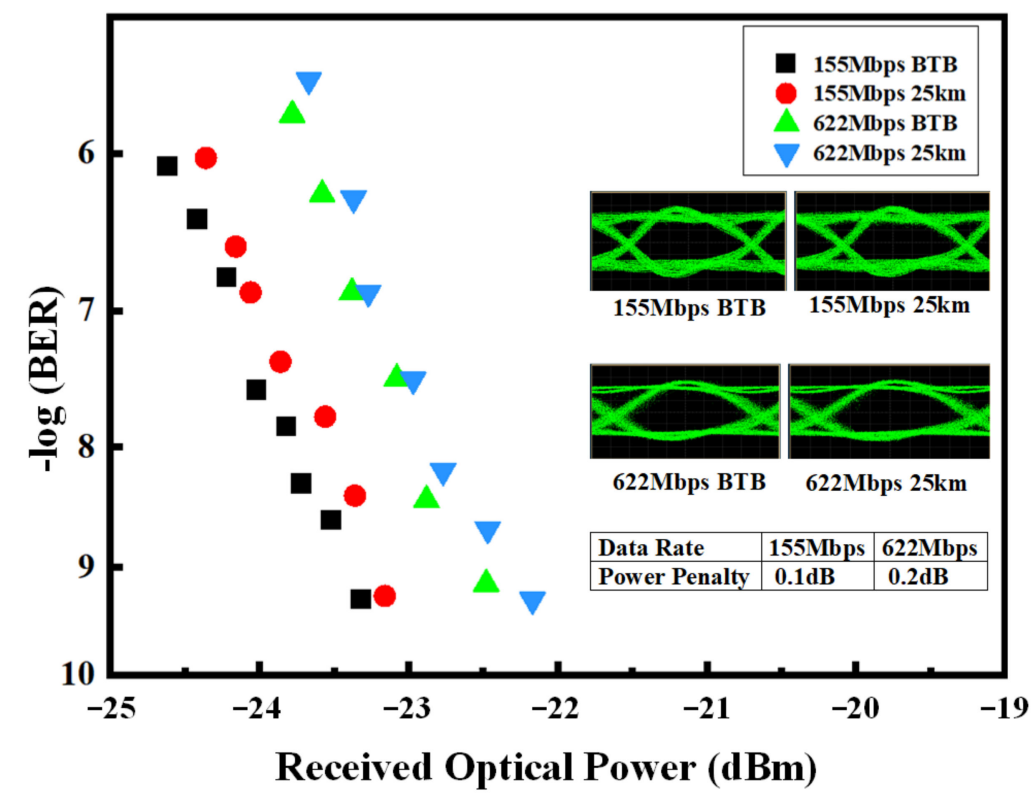

Figure 8. Comparison of BER curve and eye diagram for BTB and $25 \mathrm{~km}$ transmission.

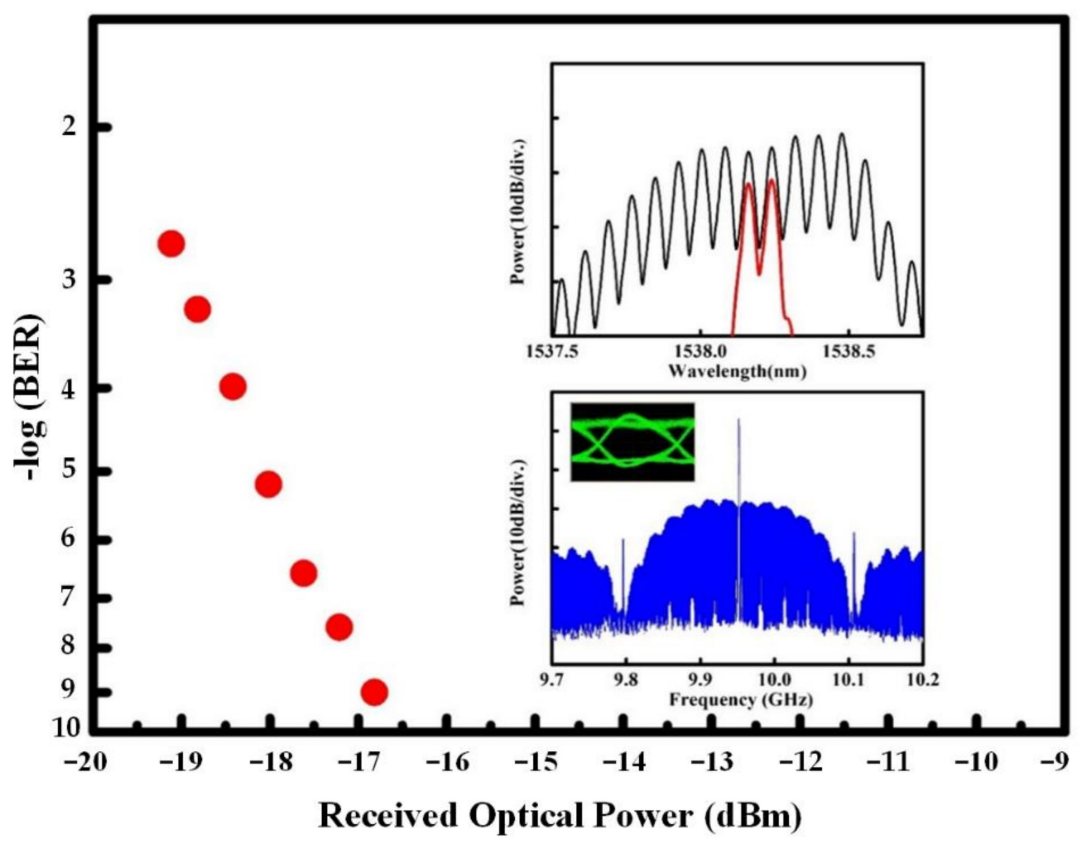

Figure 9. The measured BER, eye diagram, and electrical and optical spectrum on two selected wavelengths.

\section{Conclusions}

A self-start multi-wavelength laser source based on the OEO structure is proposed and proven in this paper. This proposed multi-wavelength laser source could be packaged in a smaller module using optical waveguide techniques to reduce power loss and simplify the complexity of OEO devices [24]. By employing the double filtering structure, the TNR value of $18 \mathrm{~dB}$ and a power fluctuation below $0.18 \mathrm{~dB}$ among 10 wavelengths are achieved. To estimate and determine the performance of the proposed scheme, OTF is deployed to select one and two wavelengths to be modulated by the input data through the RSOA. After SMF transmission, the optical signals are converted to electrical signals, which are estimated in terms of bit error rate and eye diagram. According to the experiment 
results, both one and two selected wavelengths can achieve error-free transmission and clear eye diagrams.

Author Contributions: Conceptualization, A.-M.D., R.-K.S., R.-B.C. and P.-C.P.; methodology, R.-B.C. and P.-C.P.; data preparation, R.-B.C., J.-W.L. and P.-C.P.; model validation, A.-M.D., R.-K.S. and P.-C.P.; formal analysis, Y.-C.M., H.-C.L. and P.-C.P.; investigation, A.-M.D., R.-K.S., R.-B.C., J.-W.L., Y.-C.M., H.-C.L. and P.-C.P.; and data collection and experimental setup configuration, R.-B.C. and J.-W.L. All authors have read and agreed to the published version of the manuscript.

Funding: This work was supported by the Ministry of Science and Technology, Taiwan, under Grant MOST 108-2221-E-027 -040-MY2 and by the University System of Taipei Joint Research Program, Taiwan USTP-NTUT-NTOU-105-02.

Institutional Review Board Statement: Not applicable.

Informed Consent Statement: Not applicable.

Data Availability Statement: Not applicable.

Conflicts of Interest: The authors declare no conflict of interest.

\section{References}

1. Chen, Y.W.; Zhang, R.; Hsu, C.W.; Chang, G.K. Key Enabling Technologies for the Post-5G Era: Fully Adaptive, All-Spectra Coordinated Radio Access Network with Function Decoupling. IEEE Commun. Mag. 2020, 58, 60-66. [CrossRef]

2. Shiu, R.K.; Chen, Y.W.; Peng, P.C.; Su, S.J.; Shao, G.M.; Chiu, J.; Li, J.W.; Chang, G.K. A Simplified Radio-over-Fiber System for over 100-km Long-reach n-QAM Transmission. IEEE Photonics J. 2020, 12, 7902908. [CrossRef]

3. Zhou, Q.; Chen, Y.W.; Shen, S.; Kong, Y.; Xu, M.; Zhang, J.; Chang, G.K. Proactive real-time interference avoidance in a 5G millimeter-wave over fiber mobile fronthaul using SARSA reinforcement learning. Opt. Lett. 2019, 44, 4347-4350. [CrossRef] [PubMed]

4. Chen, Y.W.; Peng, P.C.; Yan, J.H.; Shen, S.; Zhou, Q.; Huang, L.; Liu, S.; Zhang, R.; Feng, K.M.; Chang, G.K. Elastic Optical Transmission of $50 \mathrm{~Gb} / \mathrm{s} /$ Lambda OFDM Based Mobile Fronthaul via DSP-Aided Sub-band Spreading. In Proceedings of the OSA/IEEE Optical Fiber Communication Conference (OFC2019), San Diego, CA, USA, 3-7 March 2019. Paper M2B. 2.

5. Lin, C.T.; Shih, P.T.; Chen, J.; Peng, P.C.; Dai, S.P.; Jiang, W.J.; Xue, W.Q.; Chi, S. Cost-Effective Multiservices Hybrid Access Networks With no Optical Filter at Remote Nodes. IEEE Photonics Technol. Lett. 2008, 20, 812-814.

6. Chang, G.K.; Chen, Y.W. Key New Fiber Wireless Access Technologies for 5G and Beyond. IEEE 5G Tech Focus 2019, 3, 2339.

7. Alfadhli, Y.; Yao, S.; Omar, M.S.; Su, S.J.; Shen, S.; Zhang, R.; Chen, Y.W.; Peng, P.C.; Chang, G.K. Design of Flexible Fronthaul Featuring Per-UE Granularity and RU-level Puncturing for URLLC Applications. In Proceedings of the 2020 Optical Fiber Communications Conference and Exhibition (OFC), San Diego, CA, USA, 8-12 March 2020.

8. Chang, G.K.; Peng, P.C. Grand Challenges of Fiber Wireless Convergence for 5G Mobile Data Communications. In Proceedings of the 2018 23rd Opto-Electronics and Communications Conference (OECC), Jeju, Korea, 2-6 July 2018.

9. Alfadhli, Y.; Xu, M.; Liu, S.; Lu, F.; Peng, P.C.; Chang, G.K. Real-Time Demonstration of Adaptive Functional Split in 5G Flexible Mobile Fronthaul Networks. In Proceedings of the Optical Fiber Communication Conference 2018, San Diego, CA, USA, 11-15 March 2018.

10. Huang, M.Y.; Chen, Y.W.; Shiu, R.K.; Wang, H.; Chang, G.K. A Bi-Directional Multi-Band, Multi-Beam mm-Wave Beamformer for 5G Fiber Wireless Access Networks. J. Light. Technol. 2021, 39, 1116-1124. [CrossRef]

11. Alfadhli, Y.; Peng, P.C.; Shen, S.; Yao, S.; Omar, M.S.; Chen, Y.W.; Mitani, S.M.; Chang, G.K. Towards Dynamic 5G Networks Utilizing Flexible Function Split. In Proceedings of the 2019 IEEE MTT-S International Microwave Conference on Hardware and Systems for 5G and Beyond (IMC-5G), Atlanta, GA, USA, 15-16 August 2019.

12. Vallejo, L.; Ortega, B.; Almenar, V.; Bohata, J.; Zvanovec, S.; Nguyen, D.N. SOA-aided photonic signal generation for hybrid fibre and FSO 5 G transmission links. Nonlinear Opt. Its Appl. 2020, 11358, 113581R.

13. Bohata, J.; Nguyen, D.N.; Spacil, J.; Komanec, M.; Ortega, B.; Vallejo, L.; Ghassemlooy, Z.; Zvanovec, S. Experimental comparison of DSB and CS-DSB mmW formats over a hybrid fiber and FSO fronthaul network for 5G. Opt. Express 2021, 29, 27768-27782. [CrossRef] [PubMed]

14. Yao, S.; Chen, Y.W.; Su, S.J.; Alfadhli, Y.; Shen, S.; Zhang, R.; Zhou, Q.; Chang, G.K. Non-Orthogonal Uplink Services Through Co-Transport of D-RoF/A-RoF in Mobile Fronthaul. J. Light. Technol. 2020, 38, 3637-3643. [CrossRef]

15. Shiu, R.K.; Chen, Y.W.; Peng, P.C.; Wen, M.H.; Su, S.J.; Hsu, C.W.; Chang, G.K. Optical Signal Processing for W-Band Radio-OverFiber System with Tunable Frequency Response. IEEE J. Sel. Top. Quantum Electron. 2021, 27, 7600408. [CrossRef]

16. Chen, Y.W.; Chang, G.K. New radio access technologies for 5G with enhanced network reliability and channel capacity. In Proceedings of the Broadband Access Communication Technologies XIV 11307, SPIE OPTO 2020, San Francisco, CA, USA, 31 January 2020. 
17. Peng, P.C.; Shiu, R.K.; Bitew, M.A.; Chang, T.L.; Lai, C.H.; Junior, J.I. A 12 GHz wavelength spacing multi-wavelength laser source for wireless communication systems. Opt. Laser Technol. 2017, 93, 172-179. [CrossRef]

18. Lin, C.C.; Chi, Y.C.; Kuo, H.C.; Peng, P.C.; Chang-Hasnain, C.J.; Lin, G.R. Beyond-Bandwidth Electrical Pulse Modulation of a TO-Can Packaged VCSEL for 10 Gbit/s Injection-Locked NRZ-to-RZ Transmission. J. Light. Technol. 2011, 29, 830-841. [CrossRef]

19. Peng, P.C.; Feng, K.M.; Peng, W.R.; Chiou, H.Y.; Chang, C.C.; Chi, S. Long-distance fiber grating sensor system using a fiber ring laser with EDWA and SOA. Opt. Commun. 2005, 252, 127-131. [CrossRef]

20. Peng, P.C.; Lee, W.Y.; Wu, S.S.; Hu, H.L. Multiwavelength fiber laser for the fiber link monitoring system. Opt. Laser Technol. 2013, 51, 62-66. [CrossRef]

21. Yang, Z.Q.; Huang, T.J.; Chang, Y.J.; Yeh, C.H.; Chow, C.W.; Chen, J.H.; Chen, K.H. Switchable dual-wavelength singlelongitudinal-mode erbium fiber laser utilizing a dual-ring scheme with a saturable absorber. Laser Phys. 2018, $28,065104$. [CrossRef]

22. Yeh, C.H.; Chow, C.W.; Lu, S.S. Use of a reflective semiconductor optical amplifier and dual-ring architecture design to produce a stable multi-wavelength fiber laser. Laser Phys. 2014, 24, 055101. [CrossRef]

23. Chi, Y.C.; Peng, P.C.; Lin, G.-R. Clock-free RZ-BPSK data generation using a self-starting optoelectronic oscillator. J. Light. Technol. 2011, 29, 1702-1707. [CrossRef]

24. Maleki, L. The Optoelectronics Oscillator. Nat. Photonics 2011, 5, 728-730. [CrossRef]

25. Chi, Y.C.; Lin, G.R. A Q -Factor Enhanced Optoelectronic Oscillator for 40-Gbit/s Pulsed RZ-OOK Transmission. IEEE Trans. Microw. Theory Tech. 2014, 62, 3216-3223. [CrossRef] 